\title{
APPROXIMATING THE ZEROS OF ACCRETIVE OPERATORS BY THE ISHIKAWA ITERATION PROCESS
}

\author{
ZHOU HAIYUN AND JIA YUTING
}

\begin{abstract}
Some strong convergence theorems are established for the Ishikawa iteration processes for accretive operators in uniformly smooth Banach spaces.
\end{abstract}

\section{Introduction and Preliminaries}

Let $X$ be a real Banach space with a dual $X^{*}$ and normalized duality mapping $J: X \rightarrow 2^{X^{*}}$, defined by

$$
J x=\left\{f \in X^{*}:\langle f, x>=\|f\|\|x\|,\|f\|=\|x\|\},\right.
$$

where $<\cdot, \cdot>$ denotes the generalized duality pairing.

It is well known that if $X^{*}$ is strictly convex, then $J$ is single-valued and such that $J(t x)=t J x$ for all $t \geq 0, x \in X$. If $X$ is uniformly smooth, then $J$ is uniformly continuous on bounded subsets of $X$.

An operator $A$ with domain $D(A)$ and kernel $N(A)$ is said to be "accretive" if, for every $x, y \in D(A)$, there exists $j(x-y) \in J(x-y)$ such that

$$
<A x-A y, j(x-y)>\geq 0 .
$$

It is said to be "strongly accretive" if, in addition, there is a strictly increasing function $\psi: R^{+} \rightarrow R^{+}$such that $\psi(0)=0$ and

$$
<A x-A y, j(x-y)>\geq \psi(\|x-y\|)\|x-y\| .
$$

The operator $A$ is "uniformly accretive" if there is a fixed positive constant $k>0$ such that

$$
<A x-A y, j(x-y)>\geq k\|x-y\| .
$$

Furthermore, if $N(A) \neq \phi$ and the inequalities (1.1), (1.2) and (1.3) hold for any $x \in D(A)$ but $y \in N(A)$, then the corresponding operator $A$ is said

1991 Mathematics Subject Classification. Primary: 47H17.

Key words and phrases. Ishikawa iteration process, accretive operator, uniformly smooth Banach space.

Received: December 28, 1995. 
to be "quasi-accretive", "strongly quasi-accretive", and "uniformly quasiaccretive", respectively. Such operators have been extensively studied and used by various authors (see, e.g., [1]-[3]).

A quasi-accretive operator $A$ is said to satisfy "Condition (I)" if, for any $x \in D(A), p \in N(A)$, and any $j(x-p) \in J(x-p)$ the equality $<A x, j(x-$ $p)>=0$ holds if and only if $A x=A p=0$.

Recently, Xu and Roach [29] studied the characteristic conditions for the convergence of the steepest descent approximation process

$$
(*)\left\{\begin{array}{l}
x_{0} \in X \\
x_{n+1}=x_{n}-t_{n} A x_{n}, n>0
\end{array}\right.
$$

where $t_{n} \in(0, \infty), \sum_{n=0}^{\infty} t_{n}=\infty$, and $t_{n} \rightarrow 0(n \rightarrow \infty)$, for all $n \geq 0$. They proved the following two theorems.

Theorem A. ([29]) Let $X$ be a uniformly smooth Banach space and let $A: D(A)=X \rightarrow X$ be a quasi-accretive, bounded operator which satisfies the condition $(I)$. Then, for any initial value $x_{0} \in D(A)$, there are positive real numbers $T\left(x_{0}\right)$ such that the steepest descent approximation method $(*)$, with $t_{n} \leq T\left(x_{0}\right)$ for any $n$, converges strongly to a solution $x^{*}$ of the equation $A x=0$ if and only if there is a strictly increasing function $\psi: R^{+} \rightarrow$ $R^{+}, \psi(0)=0$, such that

$$
<A x_{n}-A x^{*}, J\left(x_{n}-x^{*}\right)>\geq \psi\left(\left\|x_{n}-x^{*}\right\|\right)\left\|x_{n}-x^{*}\right\| .
$$

In what follows, $F(T)$ is the fixed point set of the operator $T$.

Theorem B. ([29]) Let $X$ be a uniformly convex Banach space, $D \subset X a$ nonempty closed convex subset of $X$, and $T: D \rightarrow D$ a quasi-nonexpansive mapping (that is, $F(T) \neq \emptyset$ and $\|T x-T y\| \leq\|x-y\|$ for all $x \in D$ and $y \in F(T)$ ). Then, for any initial value $x_{0} \in D$, the Mann type iterative process

$$
\left\{\begin{array}{l}
x_{0} \in D, \\
x_{n+1}=\left(1-t_{n}\right) x_{n}+t_{n} T x_{n}, n \geq 0,0<t_{n}<1, \sum_{n=0}^{\infty} t_{n}=+\infty,
\end{array}\right.
$$

converges strongly to a fixed point $x^{*}$ of $T$ if and only if there is a strictly increasing function $f: R^{+} \rightarrow R^{+}, f(0)=0$, such that

$$
\left\|x_{n}-T x_{n}\right\| \geq f\left(d\left(x_{n}, F(T)\right), n \geq 0 .\right.
$$

One question arises naturally: Can the Ishikawa type iterative process be extended to the above theorems A and B?

In this paper we give an answer to this question.

To establish our main results, we need some special geometric properties of Banach spaces. Recall that a Banach space $X$ is said to be "uniformly convex" if $\delta_{X}(\epsilon)$, the modulus of convexity of $X$, which is defined by

$$
\delta_{X}(\epsilon)=\inf \left\{1-\frac{1}{2}\|x+y\|:\|x\|=1,\|y\|=1,\|x-y\| \geq \epsilon\right\},
$$


satisfies $\delta_{X}(0)=0$ and $\delta_{X}(\epsilon)>0$ for any $0<\epsilon \leq 2$. A Banach space $X$ is said to be "uniformly smooth" if the modulus of smoothness of $X$, defined by

$$
\rho_{X}(\tau)=\sup \left\{\frac{1}{2}\|x+y\|+\frac{1}{2}\|x-y\|-1:\|x\|=1\|y\| \leq \tau\right\}
$$

satisfies

$$
\lim _{\tau \rightarrow 0} \frac{\rho_{X}(\tau)}{\tau}=0 .
$$

It is well known that every Hilbert space $H$, the Lebesgue spaces $L^{p}(1<$ $p<\infty)$, and the Sobolev spaces $W_{m}^{p}(1<p<\infty)$ are uniformly convex and uniformly smooth.

Lemma 1.1. (Xu and Roach [28]) Let $X$ be a real uniformly smooth Banach space. Then

$$
\|x+y\|^{2} \leq\|x\|^{2}+2<y, J x>+K \max \left\{\|x\|+\|y\|, \frac{c}{2}\right\} \rho_{X}(\|y\|),
$$

for all $x, y \in X$, where $K$ and $c$ are positive constants.

Remark 1.1. In [19, p. 89] Reich established an inequality analogous to (1.5). Reich's inequality reads as follows.

Let $X$ be uniformly smooth. Then there is a continuous nondecreasing function $\beta:[0, \infty) \longrightarrow[0, \infty)$ such that $\beta(0)=0, \beta(c t) \leq c \beta(t)$ for $c \geq 1$, and

$(R I) \quad\|x+y\|^{2} \leq\|x\|^{2}+2<y, J x>+\max \{\|x\|, 1\}\|y\| \beta(\|y\|)$

for all $x$ and $y$ in $X$.

We point out that, in some sense, Reich's inequality $(R I)$ is a special case of inequality (1.5). To see this, take

$$
\beta(t)=\left\{\begin{array}{cl}
K \max \left\{1+t, \frac{c}{2}\right\} \frac{\rho_{X}(t)}{t}, & \text { if } t>0, \\
0, & \text { if } t=0 .
\end{array}\right.
$$

It is easy to verify that $\beta:[0, \infty) \longrightarrow[0, \infty)$ is a continuous nondecreasing function satisfying $\beta(a t) \leq a^{2} c_{0} \beta(t)$ for $a \geq 1$, where $c_{0}$ is a fixed positive constant. For such a function $\beta$, inequality (1.5) implies inequality $(R I)$. Since $\rho_{X}(t)$ possesses many more nice properties than $\beta(t)$, inequality (1.5) reveals much more information.

Lemma 1.2. (Xu and Roach [28]) Let X be a real uniformly convex Banach space. Then

$$
\|x+y\|^{2} \geq\|x\|^{2}+2<y, j(x)>+\sigma(x, y),
$$

where $j(x) \in J x$, and

$$
\sigma(x, y)=c \int_{0}^{1} \frac{(\|x+t y\| \vee\|x\|)^{2}}{t} \delta_{X}\left(\frac{t\|y\|}{2(\|x+t y\| \vee\|x\|)}\right) d t
$$

with c a positive constant. 
Lemma 1.3. Let $X$ be a real uniformly convex Banach space. Then

$$
\|x-y\|^{2} \leq\|x\|^{2}-2<y, j(x-y)>,
$$

for all $x, y \in X$.

Proof. Inequality (1.7) follows from the fact that the normalized duality mapping is the subdifferential of $\|x\|^{2} / 2$.

Lemma 1.4. Let $\left\{\rho_{n}\right\}_{n=0}^{\infty}$ be a nonnegative real sequence satisfying

$$
\rho_{n+1} \leq\left(1-\lambda_{n}\right) \rho_{n}+\sigma_{n}
$$

with $\lambda_{n} \in[0,1], \sum_{n=0}^{\infty} \lambda_{n}=\infty$, and $\sigma_{n}=o\left(\lambda_{n}\right)$. Then $\rho_{n} \rightarrow 0$, as $n \rightarrow \infty$.

Proof. See [20, Theorem, p. 336].

We denote by $B(0, r)$ the open ball with center at zero and radius $r>0$.

\section{Main Results}

Theorem 2.1. Let $X$ be a real uniformly smooth Banach space, and let $A: X \rightarrow X$ be a bounded quasi-accretive operator. Assume that there exists a strictly increasing and surjective function $\psi:[0, \infty)=R^{+} \rightarrow R^{+}, \psi(0)=0$, such that

$$
\left\{\begin{array}{l}
<A x_{0}-A x^{*}, J\left(x_{0}-x^{*}\right)>\geq \psi\left(\left\|x_{0}-x^{*}\right\|\right)\left\|x_{0}-x^{*}\right\| \\
<A y_{n}-A x^{*}, J\left(y_{n}-x^{*}\right)>\geq \psi\left(\left\|y_{n}-x^{*}\right\|\right)\left\|y_{n}-x^{*}\right\|
\end{array}\right.
$$

for any $x^{*} \in N(A)$, where $\left\{x_{n}\right\}_{n=0}^{\infty}$ is defined by

$$
\text { (IS) }\left\{\begin{array}{l}
x_{0} \in X, \\
x_{n+1}=x_{n}-\alpha_{n} A y_{n}-\alpha_{n} \beta_{n} A x_{n}, \\
y_{n}=x_{n}-\beta_{n} A x_{n}, n \geq 0,
\end{array}\right.
$$

where $\left\{\alpha_{n}\right\}$ and $\left\{\beta_{n}\right\}$ are two real sequences satisfying the following conditions.

(i) $0<\alpha_{n} \leq T\left(x_{0}\right)=\min \left\{\beta, \frac{\psi^{-1}\left(\left\|A x_{0}\right\|\right)}{4 M\left(x_{0}\right)}\right\}$ and $0 \leq \beta_{n} \leq T_{1}\left(x_{0}\right)=$ $\min \left\{\beta, \frac{\delta}{M\left(x_{0}\right)}, T\left(x_{0}\right)\right\}$ for all $n \geq 0$;

(ii) $\sum_{n=0}^{\infty} \alpha_{n}=\infty$;

(iii) $\alpha_{n} \rightarrow 0, \beta_{n} \rightarrow 0$ as $n \rightarrow \infty$. where

$$
\begin{gathered}
M\left(x_{0}\right)=\sup \left\{\|A y\|:\left\|y-x_{0}\right\| \leq 6 \psi^{-1}\left(\left\|A x_{0}\right\|\right)\right\}, \\
\beta=\max \left\{\beta>0: \beta^{-1} \rho_{X}\left(2 \beta M\left(x_{0}\right)\right) \leq \frac{\left\|A x_{0}\right\| \psi^{-1}\left(\left\|A x_{0}\right\|\right)}{K \max \left\{6 \psi^{-1}\left(\left\|A x_{0}\right\|\right), \frac{c}{2}\right\}}\right\},
\end{gathered}
$$

and $\delta$ is some fixed positive constant such that

$$
\|J x-J y\|<\frac{\left\|A x_{0}\right\| \psi^{-1}\left(\left\|A x_{0}\right\|\right)}{2 M\left(x_{0}\right)}
$$

whenever $\|x-y\|<\delta$ for all $x, y \in B\left(0, \psi^{-1}\left(\left\|A x_{0}\right\|\right)\right)$. 
Then the Ishikawa iteration process $\left\{x_{n}\right\}_{n=0}^{\infty}$ defined by (IS) converges strongly to a solution $x^{*}$ of the equation $A x=0$.

Proof. Since $\psi: R^{+} \rightarrow R^{+}$is strictly increasing and surjective, $\psi$ is certainly bijective. Hence $\psi^{-1}\left(\left\|A x_{0}\right\|\right)$ is well-defined. Let

$$
M\left(x_{0}\right)=\sup \left\{\|A y\|:\left\|y-x_{0}\right\| \leq 6 \psi^{-1}\left(\left\|A x_{0}\right\|\right)\right\} .
$$

Clearly, $\left\|A x_{0}\right\| \leq M\left(x_{0}\right)$. If $M\left(x_{0}\right)=0$, then $A x_{0}=0$, and, by $(I S)$, we know that $y_{n}=x_{n}=x_{0}$. From (2.1) we have

$$
0=<A x_{0}-A x^{*}, J\left(x_{0}-x^{*}\right)>\geq \psi\left(\left\|x_{0}-x^{*}\right\|\right)\left\|x_{0}-x^{*}\right\|,
$$

so that $x^{*}=x_{0}$ and hence $x_{n} \rightarrow x^{*}$ as $n \rightarrow \infty$.

Suppose that $\left\|A x_{0}\right\|>0$. Then $M\left(x_{0}\right)>0$. Since $\frac{\rho_{X}(\tau)}{\tau}$ is continuous and nondecreasing, and $\frac{\rho_{X}(\tau)}{\tau} \rightarrow 0$ as $\tau \rightarrow 0$, we can choose the largest $\beta$ such that

$$
\beta^{-1} \rho_{X}\left(2 \beta M\left(x_{0}\right)\right) \leq \frac{\psi^{-1}\left(\left\|A x_{0}\right\|\right)\left\|A x_{0}\right\|}{K \max \left\{6 \psi^{-1}\left(\left\|A x_{0}\right\|\right), \frac{c}{2}\right\}} .
$$

Let

$$
T\left(x_{0}\right)=\min \left\{\beta, \frac{\psi^{-1}\left(\left\|A x_{0}\right\|\right)}{4 M\left(x_{0}\right)}\right\} .
$$

Since $X$ is uniformly smooth, $J$ is uniformly continuous on the open ball $B\left(0, \psi^{-1}\left(\left\|A x_{0}\right\|\right)\right)$. Hence, for

$$
\epsilon_{0}=\frac{\left\|A x_{0}\right\| \psi^{-1}\left(\left\|A x_{0}\right\|\right)}{2 M\left(x_{0}\right)}>0,
$$

there is some fixed $\delta>0$ such that for all $x, y \in B\left(0, \psi^{-1}\left(\left\|A x_{0}\right\|\right)\right.$, and $\|x-y\|<\delta$, we have

$$
\|J x-J y\|<\frac{\left\|A x_{0}\right\| \psi^{-1}\left(\left\|A x_{0}\right\|\right)}{2 M\left(x_{0}\right)} .
$$

Let $T_{1}\left(x_{0}\right)=\min \left\{\beta, \frac{\delta}{M\left(x_{0}\right)}, T\left(x_{0}\right)\right\}$. We now consider two possible cases.

Case 1. There exists positive integer $n_{0}$ such that

$$
\left\|x_{n}-x^{*}\right\| \geq 2 \psi^{-1}\left(\left\|A x_{0}\right\|\right)
$$

for all $n \geq n_{0}$.

It follows from $(2.1)$ that $\left\|x_{0}-x^{*}\right\| \leq \psi^{-1}\left(\left\|A x_{0}\right\|\right)$. Without loss of generality we may assume that $\left\|x_{n_{0}-1}-x^{*}\right\|<2 \psi^{-1}\left(\left\|A x_{0}\right\|\right)$. Thus, we have

$$
\left\|x_{n_{0}-1}-x_{0}\right\| \leq\left\|x_{n_{0}-1}-x^{*}\right\|+\left\|x^{*}-x_{0}\right\|<3 \psi^{-1}\left(\left\|A x_{0}\right\|\right) .
$$

So, $\left\|A x_{n_{0}-1}\right\| \leq M\left(x_{0}\right)$. By (IS) we obtain

$$
\begin{aligned}
\left\|y_{n_{0}-1}-x^{*}\right\| & \leq\left\|x_{n_{0}-1}-x^{*}\right\|+\beta_{n_{0}-1}\left\|A x_{n_{0}-1}\right\| \\
& \leq 2 \psi^{-1}\left(\left\|A x_{0}\right\|\right)+\psi^{-1}\left(\left\|A x_{0}\right\|\right) \\
& =3 \psi^{-1}\left(\left\|A x_{0}\right\|\right) .
\end{aligned}
$$


Therefore, $\left\|y_{n_{0}-1}-x_{0}\right\| \leq 4 \psi^{-1}\left(\left\|A x_{0}\right\|\right)$ and hence $\left\|A y_{n_{0}-1}\right\| \leq M\left(x_{0}\right)$. Consequently,

$$
\begin{aligned}
\left\|x_{n_{0}}-x^{*}\right\| & \leq\left\|x_{n_{0}-1}-x^{*}\right\|+\alpha_{n_{0}-1}\left\|A y_{n_{0}-1}\right\|+\alpha_{n_{0}-1} \beta_{n_{0}-1}\left\|A x_{n_{0}-1}\right\| \\
& \leq 4 \psi^{-1}\left(\left\|A x_{0}\right\|\right),
\end{aligned}
$$

and $\left\|x_{n_{0}}-x_{0}\right\| \leq 5 \psi^{-1}\left(\left\|A x_{0}\right\|\right)$. So, $\left\|A x_{n_{0}}\right\| \leq M\left(x_{0}\right)$. Thus, we get that

$$
\begin{aligned}
\left\|y_{n_{0}}-x^{*}\right\| & \leq\left\|x_{n_{0}}-x^{*}\right\|+\beta_{n_{0}}\left\|A x_{n_{0}}\right\| \\
& \leq 5 \psi^{-1}\left(\left\|A x_{0}\right\|\right) .
\end{aligned}
$$

and $\left\|y_{n_{0}}-x_{0}\right\| \leq 6 \psi^{-1}\left(\left\|A x_{0}\right\|\right)$. Hence $\left\|A y_{n_{0}}\right\| \leq M\left(x_{0}\right)$. On the other hand,

$$
\begin{aligned}
\left\|y_{n_{0}}-x^{*}\right\| & \geq\left\|x_{n_{0}}-x^{*}\right\|-\beta_{n_{0}}\left\|A x_{n_{0}}\right\| \\
& \geq 2 \psi^{-1}\left(\left\|A x_{0}\right\|\right)-\psi^{-1}\left(\left\|A x_{0}\right\|\right) \\
& =\psi^{-1}\left(\left\|A x_{0}\right\|\right) .
\end{aligned}
$$

Since $\psi$ is increasing, we have

$$
\psi\left(\left\|y_{n_{0}}-x^{*}\right\|\right) \geq\left\|A x_{0}\right\| .
$$

Using the inequality (1.5), we obtain

$$
\begin{aligned}
& \left\|x_{n_{0}+1}-x^{*}\right\|^{2} \\
& =\left\|x_{n_{0}}-x^{*}-\alpha_{n_{0}}\left(A y_{n_{0}}-A x^{*}\right)-\alpha_{n_{0}} \beta_{n_{0}}\left(A x_{n_{0}}-A x^{*}\right)\right\|^{2} \\
& \leq\left\|x_{n_{0}}-x^{*}\right\|^{2}-2 \alpha_{n_{0}}<A y_{n_{0}}-A x^{*}, J\left(x_{n_{0}}-x^{*}\right)> \\
& +K \max \left\{\left\|x_{n_{0}}-x^{*}\right\|+\alpha_{n_{0}}\left\|A y_{n_{0}}\right\|+\alpha_{n_{0}} \beta_{n_{0}}\left\|A x_{n_{0}}\right\|, \frac{c}{2}\right\} \\
& \rho_{X} \alpha_{n_{0}}\left(\left\|A y_{n_{0}}\right\|+\beta_{n_{0}}\left\|A x_{n_{0}}\right\|\right) \\
& -2 \alpha_{n_{0}}<A y_{n_{0}}-A x^{*}, J\left(x_{n_{0}}-x^{*}\right)-J\left(y_{n_{0}}-x^{*}\right)> \\
& -2 \alpha_{n_{0}}<A y_{n_{0}}-A x^{*}, J\left(y_{n_{0}}-x^{*}\right)> \\
& +K \max \left\{6 \psi^{-1}\left(\left\|A x_{0}\right\|\right), \frac{c}{2}\right\} \rho_{X}\left(2 \alpha_{n_{0}} M\left(x_{0}\right)\right) \\
& \leq\left\|x_{n_{0}}-x^{*}\right\|^{2}+2 \alpha_{n_{0}} a_{n_{0}}-2 \alpha_{n_{0}} \psi\left(\left\|y_{n_{0}}-x^{*}\right\|\right)\left\|y_{n_{0}}-x^{*}\right\| \\
& +K \max \left\{6 \psi^{-1}\left(\left\|A x_{0}\right\|\right), \frac{c}{2}\right\} \rho_{X}\left(2 \alpha_{n_{0}} M\left(x_{0}\right)\right) \text {, }
\end{aligned}
$$

where $a_{n_{0}}=<A y_{n_{0}}-A x^{*}, J\left(x_{n_{0}}-x^{*}\right)-J\left(y_{n_{0}}-x^{*}\right)>$.

Noting that $\left\|x_{n_{0}}-y_{n_{0}}\right\| \leq \beta_{n_{0}}\left\|A x_{n_{0}}\right\|<\delta$, we know that

$$
\begin{aligned}
\left|a_{n_{0}}\right| & \leq\left\|A y_{n_{0}}\right\|\left\|J\left(x_{n_{0}}-x^{*}\right)-J\left(y_{n_{0}}-x^{*}\right)\right\| \\
& \leq M\left(x_{0}\right) \frac{\left\|A x_{0}\right\| \psi^{-1}\left(\left\|A x_{0}\right\|\right)}{2 M\left(x_{0}\right)} \\
& =\frac{1}{2}\left\|A x_{0}\right\| \psi^{-1}\left(\left\|A x_{0}\right\|\right) .
\end{aligned}
$$


Substituting (2.3) in (2.2) yields

$$
\begin{aligned}
\left\|x_{n_{0}+1}-x^{*}\right\|^{2} \leq & \left\|x_{n_{0}}-x^{*}\right\|^{2}+\alpha_{n_{0}}\left\|A x_{0}\right\| \psi^{-1}\left(\left\|A x_{0}\right\|\right) \\
& -2 \alpha_{n_{0}}\left\|A x_{0}\right\| \psi^{-1}\left(\left\|A x_{0}\right\|\right) \\
& +K \max \left\{6 \psi^{-1}\left(\left\|A x_{0}\right\|\right), \frac{c}{2}\right\} \rho_{X}\left(2 \alpha_{n_{0}} M\left(x_{0}\right)\right) \\
\leq & \left\|x_{n_{0}}-x^{*}\right\|^{2}-\alpha_{n_{0}}\left(\left\|A x_{0}\right\| \psi^{-1}\left(\left\|A x_{0}\right\|\right)\right. \\
& \left.-K \max \left\{6 \psi^{-1}\left(\left\|A x_{0}\right\|\right), \frac{c}{2}\right\} \rho_{X}\left(2 \beta M\left(x_{0}\right)\right) \beta^{-1}\right) \\
\leq & \left\|x_{n_{0}}-x^{*}\right\|^{2} .
\end{aligned}
$$

In the same way, we can prove that

$$
\left\|x_{n+1}-x^{*}\right\| \leq\left\|x_{n}-x^{*}\right\| \leq \cdots \leq\left\|x_{n_{0}}-x^{*}\right\|
$$

for all $n \geq n_{0}$. Hence $\lim _{n \rightarrow \infty}\left\|x_{n}-x^{*}\right\|$ exists, and let

$$
l=\lim _{n \rightarrow \infty}\left\|x_{n}-x^{*}\right\| .
$$

Now we want to show that $l=0$. If not, assume that $l>0$, then, by (IS), we have

$$
\begin{aligned}
l & =\lim _{n \rightarrow \infty}\left(\left\|x_{n}-x^{*}\right\|-\beta_{n}\left\|A x_{n}\right\|\right) \\
& \leq \lim _{n \rightarrow \infty} \inf \left\|y_{n}-x^{*}\right\| \leq \lim _{n \rightarrow \infty} \sup \left\|y_{n}-x^{*}\right\| \\
& \leq \lim _{n \rightarrow \infty}\left(\left\|x_{n}-x^{*}\right\|+\beta_{n}\left\|A x_{n}\right\|\right)=l .
\end{aligned}
$$

So, $\lim _{n \rightarrow \infty}\left\|y_{n}-x^{*}\right\|=l$.

We can choose positive integer $N_{1}$ such that $\left\|y_{n}-x^{*}\right\|>\frac{l}{2}$ and $\psi\left(\| y_{n}-\right.$ $\left.x^{*} \|\right)>\psi\left(\frac{l}{2}\right)$ for all $n>N_{1}$.

From (IS) we know that $\left\{A y_{n}\right\}$ is a bounded sequence.

Let $M_{1}=\sup _{n>0}\left\{\left\|A y_{n}\right\|\right\}, M_{2}=\sup _{n>0}\left\{\left\|x_{n}-x^{*}\right\|\right\}, M_{3}=\sup _{n>0}\left\{\left\|A y_{n}\right\|\right\}, M_{4}=$ $M_{1}+M_{3}$, and $M_{5}=K \max \left\{M_{2}+M_{4}, \frac{c}{2}\right\}$. Again, using the inequality (1.5), we have

$$
\begin{aligned}
\left\|x_{n+1}-x^{*}\right\|^{2} \leq & \left\|x_{n}-x^{*}\right\|^{2}-2 \alpha_{n}<A y_{n}-A x^{*}, J\left(x_{n}-x^{*}\right)> \\
& -2 \alpha_{n} \beta_{n}<A x_{n}-A x^{*}, J\left(x_{n}-x^{*}\right)> \\
& +K \max \left\{\left\|x_{n}-x^{*}\right\|+\alpha_{n}\left\|A y_{n}\right\|\right. \\
& \left.+\alpha_{n} \beta_{n}\left\|A x_{n}\right\|, \frac{c}{2}\right\} \rho_{X} \alpha_{n}\left(\left\|A y_{n}\right\|+\beta_{n}\left\|A x_{n}\right\|\right) \\
\leq & \left\|x_{n}-x^{*}\right\|^{2}+2 \alpha_{n}\left\|A y_{n}\right\|\left\|J\left(x_{n}-x^{*}\right)-J\left(y_{n}-x^{*}\right)\right\| \\
& -2 \alpha_{n} \psi\left(\left\|y_{n}-x^{*}\right\|\right)\left\|y_{n}-x^{*}\right\| \\
& +K \max \left\{M_{4}+M_{2}, \frac{c}{2}\right\} \rho_{X}\left(2 \alpha_{n} M_{4}\right) \\
\leq & \left\|x_{n}-x^{*}\right\|^{2}+2 \alpha_{n} b_{n}-\alpha_{n} l \psi\left(\frac{l}{2}\right)+M_{5} \rho_{X}\left(2 \alpha_{n} M_{4}\right) \\
= & \left\|x_{n}-x^{*}\right\|^{2}+2 \alpha_{n} b_{n}-\alpha_{n} l \psi\left(\frac{l}{2}\right)+M_{5} \rho_{X}\left(\alpha_{n} M_{4}\right),
\end{aligned}
$$


where $b_{n}=M_{1}\left\|J\left(x_{n}-x^{*}\right)-J\left(y_{n}-x^{*}\right)\right\|$. Observing that $\left\|y_{n}-x_{n}\right\|=$ $\beta_{n}\left\|A x_{n}\right\| \rightarrow 0$ as $n \rightarrow \infty$, we see that $b_{n} \rightarrow 0$ as $n \rightarrow \infty$, since $J$ is uniformly continuous on bounded sets of $X$.

At this point we choose positive integer $N_{2}$ such that

$$
2 b_{n}+\frac{M_{5} \rho_{X}\left(\alpha_{n} M_{4}\right)}{\alpha_{n}}<\frac{l}{2} \psi\left(\frac{l}{2}\right),
$$

for all $n \geq N_{2}$. Then (2.4) yields

$$
\left\|x_{n+1}-x^{*}\right\| \leq\left\|x_{n}-x^{*}\right\|^{2}-\alpha_{n} \frac{l}{2} \psi\left(\frac{l}{2}\right),
$$

thus

$$
\frac{l}{2} \psi\left(\frac{l}{2}\right) \alpha_{n} \leq\left\|x_{n}-x^{*}\right\|^{2}-\left\|x_{n+1}-x^{*}\right\|^{2}
$$

for all $n \geq N_{2}$, and hence $\frac{l}{2} \psi\left(\frac{l}{2}\right) \sum_{n=N_{2}}^{\infty} \alpha_{n} \leq\left\|x_{N_{2}}-x^{*}\right\|^{2}$, which contradicts $\sum_{n=0}^{\infty} \alpha_{n}=\infty$. So, $l=0$. From $\left(C_{1}\right)$ we see that $0 \geq 2 \psi^{-1}\left(\left\|A x_{0}\right\|\right)$. Hence $\psi^{-1}\left(\left\|A x_{0}\right\|\right)=0$ and $\left\|A x_{0}\right\|=0$, which contradicts $\left\|A x_{0}\right\|>0$. This contradiction shows that Case 1 is impossible.

Case 2. There exists an infinite subsequence $\left\{x_{n_{k}}\right\}$ of $\left\{x_{n}\right\}$ such that

$$
\left\|x_{n_{k}}-x^{*}\right\|<2 \psi^{-1}\left(\left\|A x_{0}\right\|\right) \text {. }
$$

We are going to show

$$
\left\|x_{n_{k}+m}-x^{*}\right\| \leq 2 \psi^{-1}\left(\left\|A x_{0}\right\|\right)
$$

for all positive integers $m \geq 1$.

First of all, we prove that

$$
\left\|x_{n_{k}+1}-x^{*}\right\| \leq 2 \psi^{-1}\left(\left\|A x_{0}\right\|\right)
$$

If not, $\left\|x_{n_{k}+1}-x^{*}\right\|>2 \psi^{-1}\left(\left\|A x_{0}\right\|\right)$. Observing

$$
\left\|x_{n_{k}}-x_{0}\right\| \leq\left\|x_{n_{k}}-x^{*}\right\|+\left\|x^{*}-x_{0}\right\| \leq 3 \psi^{-1}\left(\left\|A x_{0}\right\|\right),
$$

we have $\left\|A x_{n_{k}}\right\| \leq M\left(x_{0}\right)$. Hence

$$
\left\|y_{n_{k}}-x^{*}\right\| \leq\left\|x_{n_{k}}-x^{*}\right\|+\beta_{n_{k}}\left\|A x_{n_{k}}\right\| \leq 3 \psi^{-1}\left(\left\|A x_{0}\right\|\right)
$$

and

$$
\left\|y_{n_{k}}-x_{0}\right\| \leq\left\|y_{n_{k}}-x^{*}\right\|+\left\|x^{*}-x_{0}\right\| \leq 4 \psi^{-1}\left(\left\|A x_{0}\right\|\right),
$$

so that $\left.\left\|A y_{n_{k}}\right\|\right) \leq M\left(x_{0}\right)$. 
On the other hand, by (IS) we have

$$
\begin{aligned}
\left\|y_{n_{k}}-x^{*}\right\| \geq & \left\|x_{n_{k}}-x^{*}\right\|-\beta_{n_{k}}\left\|A x_{n_{k}}\right\| \\
\geq & \left\|x_{n_{k}+1}-x^{*}\right\|-\alpha_{n_{k}}\left\|A y_{n_{k}}\right\| \\
& -\beta_{n_{k}}\left\|A x_{n_{k}}\right\|-\alpha_{n_{k}} \beta_{n_{k}}\left\|A x_{n_{k}}\right\| \\
\geq & 2 \psi^{-1}\left(\left\|A x_{0}\right\|\right)-\frac{1}{4} \psi^{-1}\left(\left\|A x_{0}\right\|\right) \\
& -\frac{1}{4} \psi^{-1}\left(\left\|A x_{0}\right\|\right)-\frac{1}{2} \psi^{-1}\left(\left\|A x_{0}\right\|\right) \\
= & \psi^{-1}\left(\left\|A x_{0}\right\|\right) .
\end{aligned}
$$

Hence $\psi\left(\left\|y_{n_{k}}-x^{*}\right\|\right) \geq\left\|A x_{0}\right\|$.

Using the inequality (1.5), we get

$$
\begin{aligned}
\left\|x_{n_{k}+1}-x^{*}\right\|^{2} \leq & \left\|x_{n_{k}}-x^{*}\right\|^{2}-2 \alpha_{n_{k}}<A y_{n_{k}}-A x^{*}, J\left(x_{n_{k}}-x^{*}\right)> \\
& -2 \alpha_{n_{k}} \beta_{n_{k}}<A x_{n_{k}}-A x^{*}, J\left(x_{n_{k}}-x^{*}\right)> \\
& +K \max \left\{\left\|x_{n_{k}}-x^{*}\right\|\right. \\
& \left.+\alpha_{n_{k}}\left\|A y_{n_{k}}\right\|+\alpha_{n_{k}} \beta_{n_{k}}\left\|A x_{n_{k}}\right\|, \frac{c}{2}\right\} \rho_{X}\left(2 \alpha_{n_{k}} M\left(x_{0}\right)\right) \\
\leq & \left\|x_{n_{k}}-x^{*}\right\|^{2}+\alpha_{n_{k}}\left\|A y_{n_{k}}\right\|\left\|J\left(x_{n_{k}}-x^{*}\right)-J\left(y_{n_{k}}-x^{*}\right)\right\| \\
& -2 \alpha_{n_{k}} \psi\left(\left\|y_{n_{k}}-x^{*}\right\|\right)\left\|y_{n_{k}}-x^{*}\right\| \\
& +K \max \left\{6 \psi^{-1}\left(\left\|A x_{0}\right\|\right), \frac{c}{2}\right\} \rho_{X}\left(2 \alpha_{n_{k}} M\left(x_{0}\right)\right) \\
\leq & \left\|x_{n_{k}}-x^{*}\right\|^{2}+2 \alpha_{n_{k}} M\left(x_{0}\right) \frac{\left\|A x_{0}\right\| \psi^{-1}\left(\left\|A x_{0}\right\|\right)}{2 M\left(x_{0}\right)} \\
& -2 \alpha_{n_{k}} \psi^{-1}\left(\left\|A x_{0}\right\|\right)\left\|A x_{0}\right\| \\
& +K \max \left\{6 \psi^{-1}\left(\left\|A x_{0}\right\|\right), \frac{c}{2}\right\} \rho_{X}\left(2 \alpha_{n_{k}} M\left(x_{0}\right)\right) \\
\leq & \left\|x_{n_{k}}-x^{*}\right\|^{2}-\alpha_{n_{k}}\left(\left\|A x_{0}\right\| \psi^{-1}\left(\left\|A x_{0}\right\|\right)\right. \\
& \left.-K \max \left\{6 \psi^{-1}\left(\left\|A x_{0}\right\|\right), \frac{c}{2}\right\} \frac{\rho_{X}\left(2 \beta M\left(x_{0}\right)\right)}{\beta}\right) \\
\leq & \left\|x_{n_{k}}-x^{*}\right\|^{2} .
\end{aligned}
$$

Hence, $\left\|x_{n_{k}+1}-x^{*}\right\| \leq\left\|x_{n_{k}}-x^{*}\right\|<2 \psi^{-1}\left(\left\|A x_{0}\right\|\right)$, which is a contradiction.

By induction, we can prove that

$$
\left\|x_{n_{k}+m}-x^{*}\right\| \leq 2 \psi^{-1}\left(\left\|A x_{0}\right\|\right)
$$

for all $m \geq 1$. Hence $\left\{x_{n}\right\}$ is a bounded sequence, so are $\left\{A x_{n}\right\},\left\{y_{n}\right\}$ and $\left\{A y_{n}\right\}$.

Let $\alpha=\inf _{n>0}\left\|y_{n}-x^{*}\right\|$. Then $\alpha=0$. If not, assume that $\alpha>0$. Then $\psi\left(\left\|y_{n}-x^{*}\right\|\right) \geq \psi(\alpha)>0$. 
Again, using (1.5), we have

$$
\begin{aligned}
& \left\|x_{n+1}-x^{*}\right\|^{2} \\
& \leq\left\|x_{n}-x^{*}\right\|^{2}-2 \alpha_{n}<A y_{n}-A x^{*}, J\left(x_{n}-x^{*}\right)> \\
& -2 \alpha_{n} \beta_{n}<A x_{n}-A x^{*}, J\left(x_{n}-x^{*}\right)> \\
& +K \max \left\{\left\|x_{n}-x^{*}\right\|+\alpha_{n}\left\|A y_{n}\right\|\right. \\
& \left.+\alpha_{n} \beta_{n}\left\|A x_{n}\right\|, \frac{c}{2}\right\} \rho_{X}\left(\alpha_{n}\left(\left\|A y_{n}\right\|+\beta_{n}\left\|A x_{n}\right\|\right)\right) \\
& \leq\left\|x_{n}-x^{*}\right\|^{2}+2 \alpha_{n}\left\|A y_{n}\right\|\left\|J\left(x_{n}-x^{*}\right)-J\left(y_{n}-x^{*}\right)\right\| \\
& -2 \alpha_{n} \psi\left(\left\|y_{n}-x^{*}\right\|\right)\left\|y_{n}-x^{*}\right\| \\
& +K \max \left\{M_{4}+M_{2}, \frac{c}{2}\right\} \rho_{X}\left(2 \alpha_{n} M_{4}\right) \\
& \leq\left\|x_{n}-x^{*}\right\|^{2}+2 \alpha_{n} M_{1}\left\|J\left(x_{n}-x^{*}\right)-J\left(y_{n}-x^{*}\right)\right\| \\
& -2 \alpha_{n} \alpha \psi(\alpha)+c_{1} \rho_{X}\left(\alpha_{n}\right) \\
& \leq\left\|x_{n}-x^{*}\right\|^{2}-\alpha_{n}(2 \alpha \psi(\alpha) \\
& \left.-2 M_{1}\left\|J\left(x_{n}-x^{*}\right)-J\left(y_{n}-x^{*}\right)\right\|-c_{1} \frac{\rho_{X}\left(\alpha_{n}\right)}{\alpha_{n}}\right),
\end{aligned}
$$

where $c_{1}$ is some positive constant.

Since $\left\|J\left(x_{n}-x^{*}\right)-J\left(y_{n}-x^{*}\right)\right\| \rightarrow 0$ and $\frac{\rho_{X}\left(\alpha_{n}\right)}{\alpha_{n}} \rightarrow 0$ as $n \rightarrow \infty$, we can choose a positive integer $N_{3}$ such that

$$
2 M_{1}\left\|J\left(x_{n}-x^{*}\right)-J\left(y_{n}-x^{*}\right)\right\|+c_{1} \frac{\rho_{X}\left(\alpha_{n}\right)}{\alpha_{n}}<\alpha \psi(\alpha)
$$

for all $n \geq N_{3}$. Thus (2.5) yields

$$
\left\|x_{n+1}-x^{*}\right\|^{2} \leq\left\|x_{n}-x^{*}\right\|^{2}-\alpha \psi(\alpha) \alpha_{n}, n \geq N_{3} .
$$

Hence $\alpha \psi(\alpha) \sum_{n=N_{3}}^{\infty} \alpha_{n} \leq\left\|x_{N_{3}}-x^{*}\right\|^{2}$, which contradicts with $\sum_{n=0}^{\infty} \alpha_{n}=$ $\infty$. This contradiction shows that $\alpha=0$. Consequently, there exists an infinite subsequence $\left\{y_{n_{j}}\right\}$ of $\left\{y_{n}\right\}$ such that $y_{n_{j}} \rightarrow x^{*}$ as $j \rightarrow \infty$, and hence $x_{n_{j}} \rightarrow x^{*}$ as $j \rightarrow \infty$. As in the proof of the boundedness for $\left\{x_{n}\right\}$ we can prove that $x_{n} \rightarrow x^{*}$ as $n \rightarrow \infty$.

Remark 2.1. In the same way, as [29], we can prove that if $x_{n} \rightarrow x^{*} \in N(A)$ as $n \rightarrow \infty$ and $A: X \rightarrow X$ is quasi-accretive and satisfies condition (I), then there exists a strictly increasing function $\psi:[0, \infty) \rightarrow[0, \infty), \psi(0)=0$, such that

$$
<A y_{n}-A x^{*}, J\left(y_{n}-x^{*}\right)>\geq \psi\left(\left\|y_{n}-x^{*}\right\|\right)\left\|y_{n}-x^{*}\right\| .
$$

But, such a function $\psi$ is not surjective. From our Theorem 1, we can deduce the sufficiency of the Theorem 1 of [29]. In fact, the proof of the sufficiency of Theorem 1 of [29] has some mistakes. The authors of [29] did not require that the function $\psi$ be surjective. Since $x_{0}$ is arbitrarily chosen, it is possible 
that $\left\|A x_{0}\right\| \notin R(\psi)$ (the range of $\psi$ ). In this case, $\psi^{-1}\left(\left\|A x_{0}\right\|\right)$ is not well defined.

Remark 2.2. From our Theorem 1, we can deduce the relevant results of Tan and $\mathrm{Xu}[23]$ and Chidume [6-11].

Remark 2.3. We would like to point out that Theorem 2.1 is closely related to the well known strong convergence theorems in [4], [18]. Although they can't be deduced directly from Theorem 2.1, we can yield those results with our new approach. The detailed discussion of the relationship between Theorem 2.1 and the corresponding strong convergence theorems will be presented in a subsequent paper.

In the sequel, we prove the convergence theorems of the Ishikawa iteration processes for quasi-nonexpansive operators.

Let $C$ be a nonempty bounded closed convex subset of Banach space $X$. An operator $T: C \rightarrow C$ is said to be quasi-nonexpansive, if the fixed point set $F(T)$ of $T$ is nonempty, and

$$
\|T x-T y\| \leq\|x-y\|
$$

for all $x \in C$ but $y \in F(T)$.

The operator $T$ is said to satisfy "Condition (A)" if there is a nondecreasing function $f: R^{+} \rightarrow R^{+}, f(0)=0, f(r)>0$ for all $r>0$, such that

$$
\|x-T x\| \geq f(d(x, F(T))), x \in C,
$$

where $d(x, F(T))=\inf \{\|x-z\|: z \in F(T)\}$ (see, e.g., [16]).

We study the following Ishikawa iteration process:

$$
\left(\mathrm{I}^{\prime}\right)\left\{\begin{array}{l}
x_{0} \in C \\
x_{n+1}=\left(1-\alpha_{n}\right) x_{n}+\alpha_{n} T y_{n} \\
y_{n}=\left(1-\beta_{n}\right) x_{n}+\beta_{n} T x_{n}, n>0
\end{array}\right.
$$

where $\left\{\alpha_{n}\right\}$ and $\left\{\beta_{n}\right\}$ are real sequences satisfying:

(i) $0<\alpha_{n}, \beta_{n}<1$;

(ii) $\sum_{n=0}^{\infty} \alpha_{n}=\infty$;

(iii) $\alpha_{n} \rightarrow 0, \beta_{n} \rightarrow 0$ as $n \rightarrow \infty$.

Let $A=I-T$. Then (I') yields

$$
\left(\mathrm{I}^{\prime \prime}\right)\left\{\begin{array}{l}
x_{0} \in C, \\
x_{n+1}=x_{n}-\alpha_{n} A y_{n}-\alpha_{n} \beta_{n} A x_{n}, \\
y_{n}=x_{n}-\beta_{n} A x_{n}, n>0 .
\end{array}\right.
$$

We also need the following lemmas.

Lemma 2.1. For any $y \in F(T), \lim _{n \rightarrow \infty}\left\|x_{n}-y\right\|$ exists. 
Proof. From $\left(I^{\prime}\right)$, we have

$$
\begin{aligned}
\left\|x_{n+1}-y\right\| & \leq\left(1-\alpha_{n}\right)\left\|x_{n}-y\right\|+\alpha_{n}\left\|y_{n}-y\right\| \\
& \leq\left(1-\alpha_{n}\right)\left\|x_{n}-y\right\|+\alpha_{n}\left(\left(1-\beta_{n}\right)\left\|x_{n}-y\right\|+\beta_{n}\left\|T x_{n}-T y\right\|\right) \\
& \leq\left(1-\alpha_{n}\right)\left\|x_{n}-y\right\|+\alpha_{n}\left\|x_{n}-y\right\| \\
& =\left\|x_{n}-y\right\| .
\end{aligned}
$$

Lemma 2.2. (Xu and Roach [29]) Let $X$ be a real uniformly convex Banach space and let $C$ be a nonempty closed convex subset of $X$. Assume $T: C \rightarrow C$ is quasi-nonexpansive. Let $A=I-T$. Then

$$
<A x-A y, j(x-y)>\geq \frac{c}{2}\|x-y\|^{2} \int_{0}^{\frac{\|A x-A y\|}{2\|x-y\|}} \frac{\delta_{X}(\epsilon)}{\epsilon} d \epsilon,
$$

where $j(x-y) \in J(x-y), \delta_{X}(\epsilon)$ is the modulus of convexity of $X$, and $c$ is a fixed positive constant.

Now we prove:

Theorem 2.2. Let $X$ be a real uniformly convex Banach space, and let $C$ be a nonempty bounded closed convex subset of $X$. Assume that $T: C \rightarrow C$ is quasi-nonexpansive. If $T$ satisfies the condition $(A)$, then the Ishikawa type iteration sequence $\left\{x_{n}\right\}_{n=0}^{\infty}$ defined by $\left(I^{\prime \prime}\right)$ converges strongly to some fixed point of $T$.

Proof. We consider the following two possible cases.

Case a. $\inf _{n>0}\left\|x_{n}-y\right\|=0, y \in F(T)$.

In this case, there exists subsequence $\left\{x_{n_{j}}\right\}$ of $\left\{x_{n}\right\}$ such that $x_{n_{j}} \rightarrow y$ as $j \rightarrow \infty$. By Lemma 2.1, we know that $x_{n} \rightarrow y$ as $n \rightarrow \infty$.

Case b. $\inf _{n>0}\left\|x_{n}-y\right\|=\alpha>0, y \in F(T)$.

In this case, we again consider two possible cases.

Case $\mathrm{b}_{1} \cdot \inf _{n>0}\left\|A x_{n}\right\|=0$.

In this case, there exists subsequence $\left\{A x_{n_{j}}\right\}$ of $\left\{A x_{n}\right\}$ such that $A x_{n_{j}} \rightarrow 0$ as $j \rightarrow \infty$. Since $T$ satisfies the condition (A) we have

$$
\left\|A x_{n_{j}}\right\|=\left\|x_{n_{j}}-T x_{n_{j}}\right\| \geq f\left(d\left(x_{n_{j}}, F(T)\right) .\right.
$$

Hence $f\left(d\left(x_{n_{j}}, F(T)\right) \rightarrow 0\right.$ as $j \rightarrow \infty$.

Since $f: R^{+} \rightarrow R^{+}$is nondecreasing and $f(r)>0$ for all $r>0$, so, $d\left(x_{n_{j}}, F(T)\right) \rightarrow 0$ as $j \rightarrow \infty$. By Lemma 2.1, we see that $\lim _{n \rightarrow \infty} d\left(x_{n}, F(T)\right)$ exists. Hence $d\left(x_{n}, F(T)\right) \rightarrow 0$.

At this point we can choose a subsequence $\left\{x_{n_{k}}\right\}$ of $\left\{x_{n}\right\}$ and $\left\{p_{k}\right\} \subset F(T)$, respectively, such that

$$
\left\|x_{n_{k}}-p_{k}\right\|<2^{-k} \text { for all } k \geq 1 .
$$

By Lemma 2.1, we see that

$$
\left\|x_{n_{k+1}}-p_{k}\right\| \leq\left\|x_{n_{k}}-p_{k}\right\|<2^{-k},
$$


so that

$$
\begin{aligned}
\left\|p_{k+1}-p_{k}\right\| & \leq\left\|x_{n_{k+1}}-p_{k+1}\right\|+\left\|x_{n_{k+1}}-p_{k}\right\| \\
& \leq 2^{-(k+1)}+2^{-k} \\
& <2^{-k+1}
\end{aligned}
$$

for all $k \geq 1$. Hence $\left\{p_{k}\right\}$ must be a Cauchy sequence. Thus we can assume $p_{k} \rightarrow p$ as $k \rightarrow \infty$.

Since $F(T)$ is closed, we know that $p \in F(T)$. Therefore, $x_{n_{k}} \rightarrow p$ as $k \rightarrow \infty$ and hence $x_{n} \rightarrow p$ as $n \rightarrow \infty$, since $\lim _{n \rightarrow \infty}\left\|x_{n}-p\right\|$ exists.

Case $\mathrm{b}_{2} \cdot \inf _{n>0}\left\|A x_{n}\right\|=r>0$.

Let $Q=\sup _{n>0}\left\|x_{n}-y\right\|>0, y \in F(T)$. Then

$$
0<\frac{r}{2 Q} \leq \frac{\left\|A x_{n}-A y\right\|}{2\left\|x_{n}-y\right\|} \leq 1 .
$$

Applying Lemma 2.2 we obtain

$$
\begin{aligned}
<A x_{n}-A y, j\left(x_{n}-y\right)> & \geq \frac{c}{2}\left\|x_{n}-y\right\|^{2} \int_{0}^{\frac{r}{2 Q}} \frac{\delta_{X}(\epsilon)}{\epsilon} d \epsilon \\
& \geq k\left\|x_{n}-y\right\|^{2},
\end{aligned}
$$

where

$$
0<k<\min \left\{1, \frac{c}{2} \int_{0}^{\frac{r}{2 Q}} \frac{\delta_{X}(\epsilon)}{\epsilon} d \epsilon\right\} .
$$

Using Lemma 1.3, $\left(I^{\prime \prime}\right)$ and (2.6) we have

$$
\begin{aligned}
& \left\|x_{n+1}-y\right\|^{2} \\
& \leq\left\|x_{n}-y\right\|^{2}-2 \alpha_{n}<A y_{n}-A y, j\left(x_{n+1}-y\right)> \\
& \quad-2 \alpha_{n} \beta_{n}<A x_{n}, j\left(x_{n+1}-y\right)> \\
& \leq\left\|x_{n}-y\right\|^{2}-2 \alpha_{n}<A y_{n}-A x_{n+1}, j\left(x_{n+1}-y\right)> \\
& \quad-2 \alpha_{n} \beta_{n}<A x_{n}, j\left(x_{n+1}-y\right)> \\
& \quad-2 \alpha_{n}<A x_{n+1}-A y, j\left(x_{n+1}-y\right)> \\
& \quad-2 \alpha_{n} \beta_{n}<A x_{n}, j\left(x_{n+1}-y\right)> \\
& \leq \\
& \quad\left\|x_{n}-y\right\|^{2}-2 \alpha_{n} c_{n}-2 \alpha_{n} k\left\|x_{n+1}-y\right\|^{2} \\
& \quad-2 \alpha_{n} \beta_{n}<A x_{n}, j\left(x_{n+1}-y\right)>,
\end{aligned}
$$

where $c_{n}=<A y_{n}-A x_{n+1}, j\left(x_{n+1}-y\right)>$.

We show $c_{n} \rightarrow 0$ as $n \rightarrow \infty$. Indeed, since

$$
\begin{aligned}
\| A y_{n} & -A x_{n+1} \| \\
\leq & 2\left\|y_{n}-x_{n+1}\right\| \\
\leq & 2 \beta_{n}\left\|A x_{n}\right\|+2 \alpha_{n}\left\|A y_{n}\right\|+2 \alpha_{n} \beta_{n}\left\|A x_{n}\right\| \\
& \rightarrow 0 \text { as } n \rightarrow \infty,
\end{aligned}
$$


and $\left\|j\left(x_{n+1}-y\right)\right\|=\left\|x_{n+1}-y\right\|$ is bounded, $c_{n} \rightarrow 0$ as $n \rightarrow \infty$. Let $d_{n}=<A x_{n}, j\left(x_{n+1}-y\right)>$ and

$$
\sigma_{n}=\frac{-2 \alpha_{n}\left(c_{n}+\beta_{n} d_{n}\right)}{1+2 k \alpha_{n}} .
$$

From (2.7) we get

$$
\begin{aligned}
\left\|x_{n+1}-y\right\|^{2} & \leq \frac{1}{1+2 k \alpha_{n}}\left\|x_{n}-y\right\|^{2}+\sigma_{n} \\
& =\left(1-2 k \alpha_{n}+\frac{4 k^{2} \alpha_{n}^{2}}{1+2 k \alpha_{n}}\right)\left\|x_{n}-y\right\|^{2}+\sigma_{n} \\
& \leq\left(1-k \alpha_{n}\right)\left\|x_{n}-y\right\|^{2}+\sigma_{n} .
\end{aligned}
$$

Set $\rho_{n}=\left\|x_{n}-y\right\|^{2}, \lambda_{n}=k \alpha_{n}$. Then, $\lambda_{n} \in[0,1], \sum_{n=0}^{\infty} \lambda_{n}=\infty$, and $\sigma_{n}=$ $o\left(\lambda_{n}\right)$. By Lemma 1.4, we see that $\rho_{n} \rightarrow 0$ as $n \rightarrow \infty$, i.e., $x_{n} \rightarrow y$ as $n \rightarrow \infty$, which contradicts with $\inf _{n>0}\left\|x_{n}-y\right\|=\alpha>0$.

From the above discussion, we know that $\left\{x_{n}\right\}$ converges strongly to some fixed point of $T$.

In the same way, we can prove

Theorem 2.3. Let $X$ and $C$ be as in Theorem 2.2, and let $T: C \rightarrow C$ be a quasi-nonexpansion with $T(C)$ compact. Then the conclusion of Theorem 2.2 is still true.

Acknowledgment. The authors wish to thank the referee for many valuable suggestions.

\section{REFERENCES}

[1] F. E. Browder, Nonlinear operators and nonlinear equations of evolution in Banach Spaces, Proc. Sympos. Pure Math. 18, Amer. Math. Soc, Providence, Rhode Island, 1976.

[2] F. E. Browder and W. V. Petryshyn, Construction of fixed points of nonlinear mappings in Hilbert space, J. Math. Anal. Appl. 20 (1967), 197-228.

[3] R. E. Bruck, The iterative solution of the equation $y \in x+T x$ for a monotone operator $T$ in Hilbert space, Bull. Amer. Math. Soc. 9 (1973), 1258-1262.

[4] R. E. Bruck and S. Reich, A general convergence principle in nonlinear functional Analysis, Nonl. Anal. TMA 4 (1980), 939-950.

[5] C. E. Chidume, The iterative solution of the equation $f \in x+T x$ for a monotone operator T in $L_{p}$ spaces, J. Math. Anal. Appl. 116 (1986), 531-537.

[6] C. E. Chidume, Iterative approximation of fixed points of Lipschitzian strictly pseudo-contractive mappings, Proc. Amer. Math. Soc. 99 (1987), 283-288.

[7] C. E. Chidume, Iterative solution of nonlinear equations of the monotone and dissipative type, Appl. Anal. 13 (1989), 79-86.

[8] C. E. Chidume, Iterative solution of nonlinear equations of monotone type in Banach spaces, Bull. Austral. Math. Soc. 42 (1990), 21-31.

[9] C. E. Chidume, An iterative process for nonlinear Lipschitzian strongly accretive operators in $L_{P}$ space, J. Math. Anal. Appl. 151 (1990), 453-461.

[10] C. E. Chidume, Approximation of fixed point of strongly pseudocontractive mappings, Proc. Amer. Math. Soc. 120 (1994), 545-551. 
[11] C. E. Chidume, Iterative solution of nonlinear equations with strongly accretive operators, J. Math. Anal. Appl. 192 (1995), 502-518.

[12] L. Deng, On Chidume's open questions, J. Math. Anal. Appl. 174 (1993), 441-449.

[13] L. Deng and X. P. Ding, Iterative approximation of Lipschitz strictly pseudocontractive mappings in uniformly smooth Banach spaces, Nonl. Anal. TMA 24 (1995), 981-987.

[14] J. Gwinner, On the convergence of some iteration process in uniformly convex Banach spaces, Proc. Amer. Math. Soc. 81 (1978), 29-35.

[15] S. Ishikawa, Fixed points by a new iteration method, Proc. Amer. Math. Soc. 44 (1974), 147-150.

[16] S. Ishikawa, Fixed points and iteration of a nonexpansive mapping in a Banach space, Proc. Amer. Math. Soc. 59 (1976), 65-71.

[17] W. R. Mann, Mean value methods in iteration, Proc. Amer. Math. Soc. 4 (1953), 506-510.

[18] O. Nevanlinna and S. Reich, Strong convergence of contraction semigroups and of iterative methods for accretive operators in Banach spaces, Israel J. Math. 32 (1979), 44-58.

[19] S. Reich, An iterative procedure for constructing zeros of accretive sets in Banach space, Nonl. Anal. TMA 2 (1978), 85-92.

[20] S. Reich, Constructive techniques for accretive and monotone operators, in Applied Nonlinear Analysis, Academic Press, New York, 1979, 335-345.

[21] B. E. Rhoades, Comments on two fixed point iteration methods, J. Math. Anal. Appl. 56 (1976), 741-750.

[22] B. E. Rhoades, Fixed point iterations for certain nonlinear mappings, J. Math. Anal. Appl. 183 (1994), 118-120.

[23] K. K. Tan and H. K. Xu, Iterative solutions to nonlinear equations of strongly accretive operators in Banach space, J. Math. Anal. Appl. 178 (1993), 9-12.

[24] K. K. Tan and H. K. Xu, Approximating fixed points of nonexpansive mappings by the Ishikawa iteration process, J. Math. Anal. Appl. 178 (1993), 301-308.

[25] K. K. Tan and H. K. Xu, Fixed point iteration processes for asymptotically nonexpansive mappings, Proc. Amer. Math. Soc. 122 (1994), 733-739.

[26] X. L. Weng, Fixed point iteration for local strongly pseudo-contractive mapping, Proc. Amer. Math. Soc. 113 (1991), 727-731.

[27] H. K. Xu, Inequalities in Banach spaces with applications, Nonl. Anal. TMA 16 (1991), 1127-1138.

[28] Z. Xu and G. F. Roach, Characteristic inequalities of uniformly convex and uniformly smooth Banach spaces, J. Math. Anal. Appl. 157 (1991), 189-210.

[29] Z. Xu and G. F. Roach , A necessary and sufficient condition for convergence of steepest descent approximation to accretive operator equations, J. Math. Anal. Appl. 167 (1992), 340-354.

[30] Z. You and Z. Xu, A class of iteration methods for a strongly monotonic operator equation and application to finite element approximate solution of nonlinear elliptic boundary value problem, J. Comput. Math. 2 (1984), 112-121.

[31] H. Zhou and Y. Jia, Approximation of fixed point of strongly pseudo-contractive mappings without Lipschitz assumption, Proc. Amer. Math. Soc. (to appear).

Department of Mathematics, Shijiazhuang Engineering College, Shijiazhuang 050003, People's Republic of China

Department of Mathematics, Hebei Teachers University, Shijiazhuang 050016, People's Republic of China 


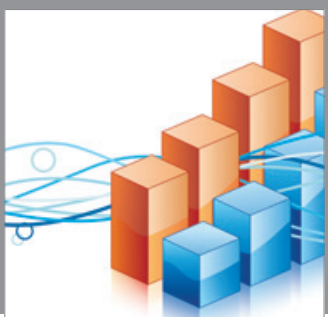

Advances in

Operations Research

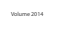

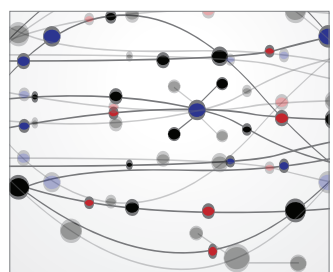

\section{The Scientific} World Journal
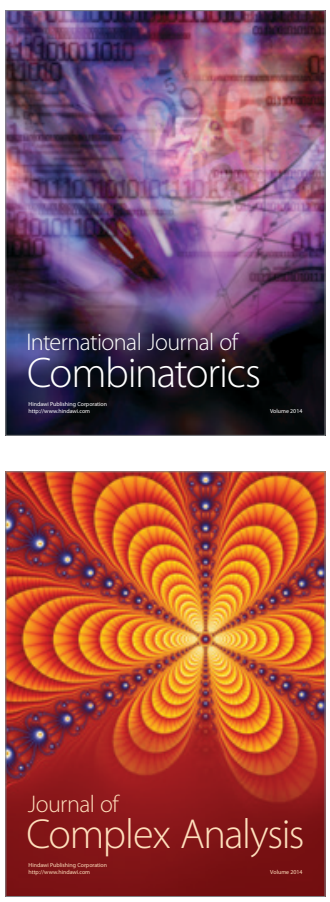

International Journal of

Mathematics and

Mathematical

Sciences
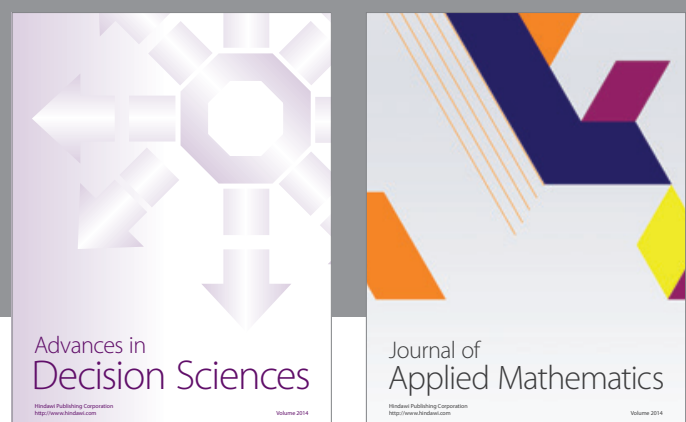

Journal of

Applied Mathematics
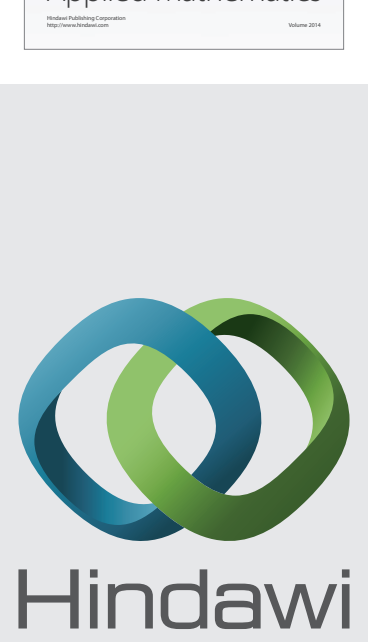

Submit your manuscripts at http://www.hindawi.com
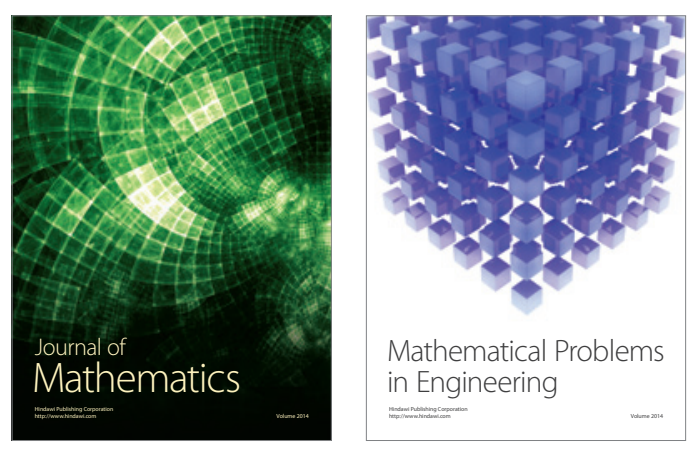

Mathematical Problems in Engineering
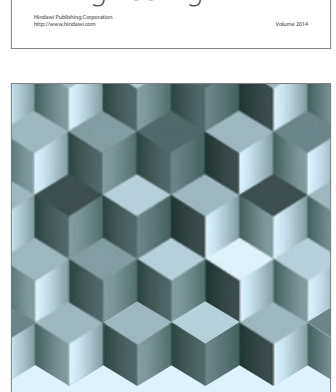

Journal of

Function Spaces
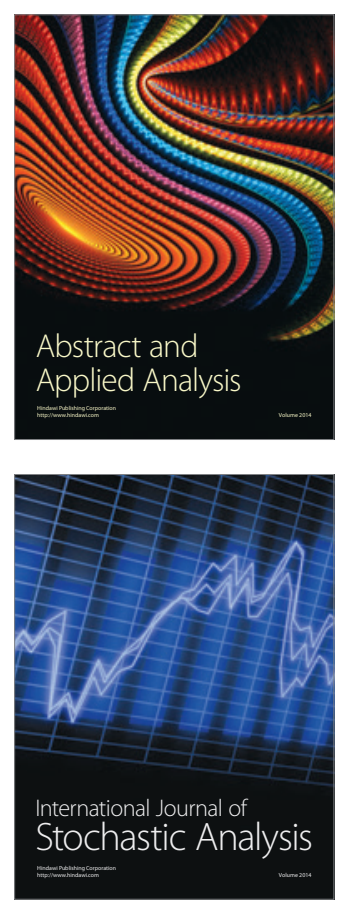

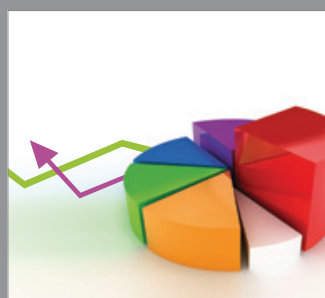

ournal of

Probability and Statistics

Promensencen
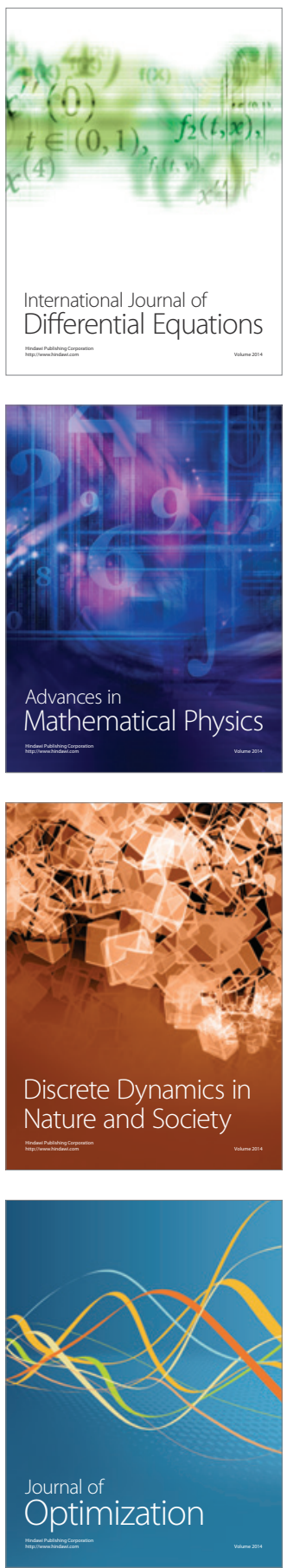